\title{
Classifying lower grade glioma cases according to whole genome gene expression
}

\author{
Baoshi Chen ${ }^{1, *}$, Tingyu Liang ${ }^{1,2, *}$, Pei Yang ${ }^{1,2,3, *}$, Haoyuan Wang ${ }^{4}$, Yanwei Liu ${ }^{1,2,3}$, \\ Fan Yang ${ }^{1,2}$, Gan You'1,2,3 \\ ${ }^{1}$ Department of Neurosurgery, Beijing Tiantan Hospital, Capital Medical University, Beijing, China \\ ${ }^{2}$ Beijing Neurosurgical Institute, Capital Medical University, Beijing, China \\ ${ }^{3}$ Chinese Glioma Cooperative Group (CGCG), China \\ ${ }^{4}$ Department of Neurosurgery, Guangdong Zhujiang Hospital, Southern Medical University, Guangzhou, China \\ *These authors have contributed equally to this work \\ Correspondence to: Gan You, email: Ganyou2016@126.com
}

Keywords: lower grade glioma, risk score, gene signature, prognosis

Received: February 17, $2016 \quad$ Accepted: August 13, 2016

Published: September 22, 2016

\section{ABSTRACT}

OBJECTIVE: To identify a gene-based signature as a novel prognostic model in lower grade gliomas.

RESULTS: A gene signature developed from HOXA7, SLC2A4RG and MN1 could segregate patients into low and high risk score groups with different overall survival (OS), and was validated in TCGA RNA-seq and GSE16011 mRNA array datasets. Receiver operating characteristic (ROC) was performed to show that the three-gene signature was more sensitive and specific than histology, grade, age, IDH1 mutation and 1p/19q co-deletion. Gene Set Enrichment Analysis (GSEA) and GO analysis showed high-risk samples were associated with tumor associated macrophages (TAMs) and highly invasive phenotypes. Moreover, HOXA7-siRNA inhibited migration and invasion in vitro, and downregulated MMP9 at the protein level in U251 glioma cells.

METHODS: A cohort of 164 glioma specimens from the Chinese Glioma Genome Atlas (CGGA) array database were assessed as the training group. TCGA RNA-seq and GSE16011 mRNA array datasets were used for validation. Regression analyses and linear risk score assessment were performed for the identification of the three-gene signature comprising HOXA7, SLC2A4RG and MN1.

CONCLUSIONS: We established a three-gene signature for lower grade gliomas, which could independently predict overall survival (OS) of lower grade glioma patients with higher sensitivity and specificity compared with other clinical characteristics. These findings indicate that the three-gene signature is a new prognostic model that could provide improved OS prediction and accurate therapies for lower grade glioma patients.

\section{INTRODUCTION}

Gliomas are highly malignant intracranial tumors that account for more than half of primary central nerves system (CNS) cancers [1]. Despite the recent progress in neurosurgery, radiotherapy and chemotherapy, no great improvement of overall survival (OS) in gliomas patients has been reported in the past four decades [2].

Gliomas could be divided into four grades (I-IV) according to the World Health Organization (WHO) [3]. WHO grades II and III, including astrocytomas, oligodendrogliomas and mixed oligoastrocytomas are considered lower grade gliomas [4]. The common morphology-based classification could yield subjective differences regarding histological typing and grading between observers. In neuro-oncological practice, no clear national consensus for the diagnosis of adult gliomas has been reached [5], because of biological and clinical heterogeneity. Indeed, survival times in lower grade patients is totally different; some of them lived for more than 5 years in the CGGA array database, while others had really poor survival (less than 1 year).

With precision medicine becoming more popular, it would be helpful to lower grade gliomas patients to obtain a more accurate OS prediction and effective 
treatment [6]. With the development of high-throughput technologies, multiple biomarkers have been identified for predicting clinical results of cancer patients $[7,8]$. In some malignant tumors, such as lung cancer and breast cancer, gene expression profiles of primary tumors have been established to predict OS [9, 10]. Many genes such as isocitrate dehydrogenase1 mutation (IDH1mut), epidermal growth factor receptor (EGFR) amplification, and Ki67expression have been included in gliomas to predict prognosis $[4,11,12]$. With deeper understanding of gliomas, it is acknowledged that polygenic abnormity, rather than single gene alterations, causes gliomas [13]. Here, we evaluated gene microarray expression profiles of three independent cohorts to establish a three-gene (HOXA7, SLC2A4RG and MN1) signature model. Using the new prognostic model, OS of lower grade gliomas patients could be predicted more objectively and accurately. Patients with different risk scores had various clinical and molecular features. The three-gene signature may have clinical implications in treating gliomas.

\section{RESULTS}

\section{Discovery of the three-gene signature and prediction of survival in the CGGA database}

In 164 CGGA lower grade gliomas samples, there was significant expression difference $(p<0.05)$ in 28 genes (Figure $1 p<0.05)$ between short survival time $(<$
1 year) and long survival time ( $>5$ years). Then, Cox regression was carried out for analyzing genes in the training set (CGGA set); as a result, a total of 9 genes (CADM2, CEP68, CNRIP1, HOXA7, MBOAT2, MN1, SLC2A4RG, TBC1D5, TRAPPC2P1) were correlated with OS in grades II and III samples. For further assessment, univariate Cox regression was performed for the TCGA RNA-seq and GSE16011 mRNA array databases. Three genes (HOXA7, SLC2A4RG and MN1) were selected to establish a three-gene signature in lower grade gliomas (Table 1). Based on the three genes, we carried out the risk score assessment for predicting OS in lower grade gliomas [14]. In the training cohort, using the three-gene signature, we could divide lower grade glioma patients into low- and high-risk groups; a median risk score of -1.06 was chosen as cut off value for lower grade gliomas. Median survival times in the low- and high-risk groups were 1980 and 1610 days, respectively. High-risk group individuals showed significantly lower OS compared with low-risk group counterparts $(p<0.05$ Figure 2A); in addition, survival was markedly reduced in the high-risk group (Supplementary Table S1).

\section{Validation of the use fullness of the signature in two additional datasets}

To verify the accuracy of the above results, TCGA RNA-seq and GSE 16011 array datasets were selected for assessment. The clinical characteristics of the two datasets

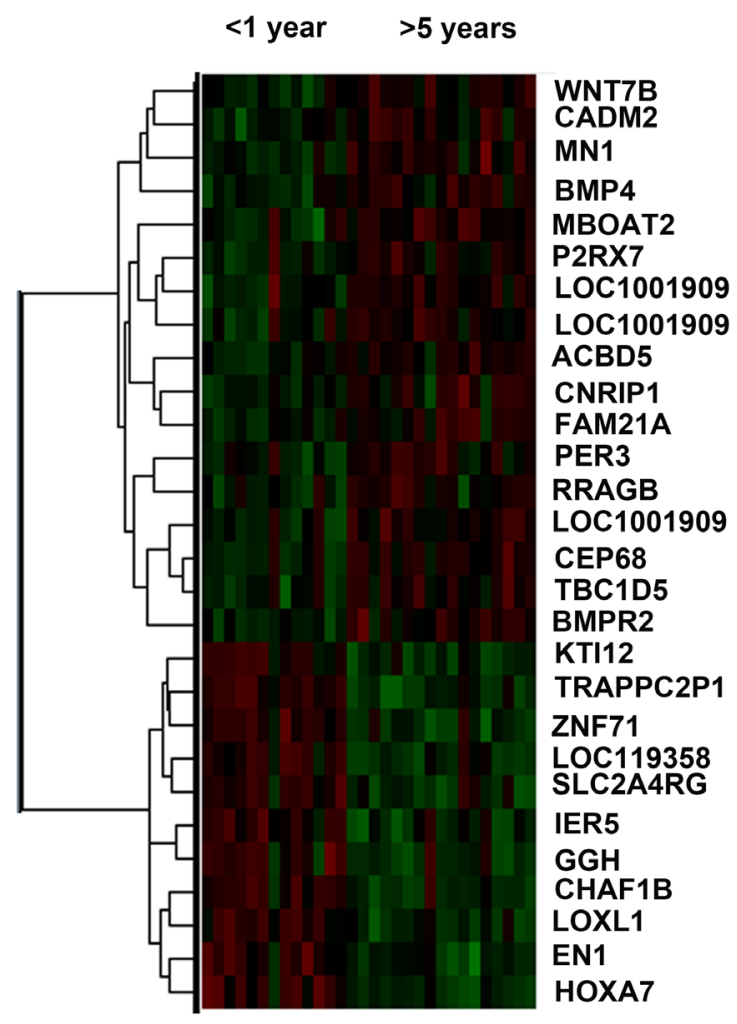

Figure 1: Heat map of differentially expressed genes in short (<1year) and long ( $>5 y e a r)$ survival groups. Red, High expression; Green, Low expression. 
Table 1: Three genes associated significantly with overall survival (OS)

\begin{tabular}{lcccc}
\hline Gene & HR & $\mathbf{9 5 \%}$ CI & B & P \\
\hline MN1 & 0.378 & $0.270-0.528$ & -0.974 & $<0.0001$ \\
HOXA7 & 1.379 & $1.260-1.509$ & 0.321 & $<0.0001$ \\
SLC2A4RG & 2.539 & $1.904-3.386$ & 0.931 & $<0.0001$ \\
\hline
\end{tabular}
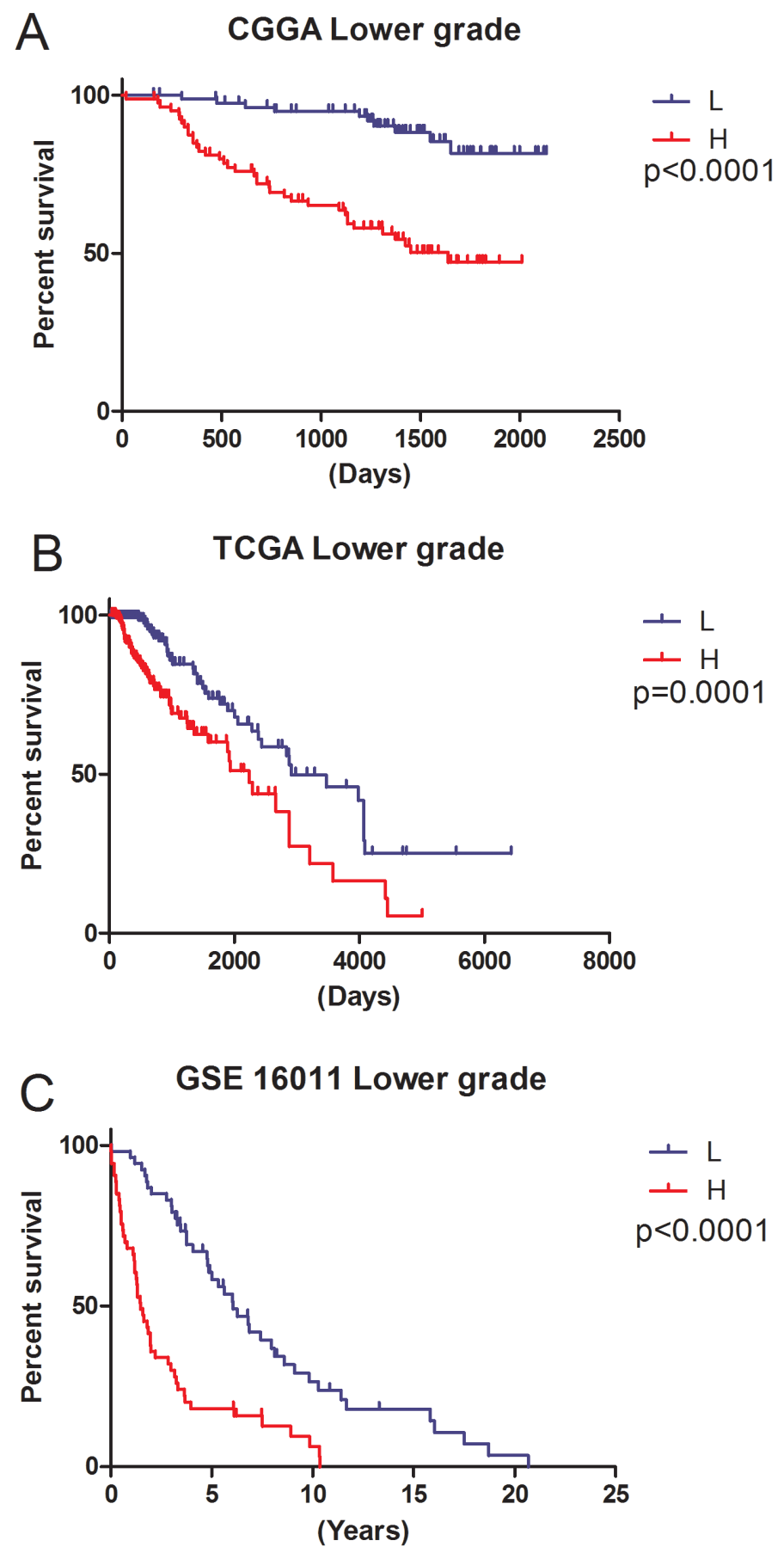

Figure 2: Ability of the three-gene signature to predict overall survival in the training and validation sets. L, Low-risk group; H, High-risk group. A. CGGA mRNA array data $(\mathrm{p}<0.0001)$; B. TCGA RNA-seq data $(\mathrm{p}<0.0001)$; C. GSE16011 mRNA array data $(\mathrm{p}<0.0001)$. 
Table 2: Characteristics of patients in low risk and high-risk group in CGGA dataset

\begin{tabular}{|c|c|c|c|c|c|}
\hline \multirow{3}{*}{ Clinical factors } & & \multicolumn{3}{|c|}{ Lower grade } & \multirow{3}{*}{$\mathbf{P}$} \\
\hline & & total & LR & HR & \\
\hline & & 164 & 82 & 82 & \\
\hline \multirow{3}{*}{ Age } & $\leq 39$ & 88 & 45 & 43 & $>0.05$ \\
\hline & $>39$ & 76 & 37 & 39 & \\
\hline & NA & 0 & & & \\
\hline \multirow{3}{*}{ Gender } & $\mathrm{F}$ & 71 & 33 & 38 & $>0.05$ \\
\hline & M & 93 & 49 & 44 & \\
\hline & NA & 0 & & & \\
\hline \multirow{3}{*}{ Grade } & $\mathrm{G} 2$ & 117 & 72 & 45 & $<0.0001$ \\
\hline & G3 & 47 & 10 & 37 & \\
\hline & NA & 0 & & & \\
\hline \multirow{3}{*}{ IDH1 mut } & MUT & 104 & 57 & 47 & $>0.05$ \\
\hline & WT & 56 & 23 & 33 & \\
\hline & NA & 4 & & & \\
\hline \multirow{3}{*}{ TP53 } & MUT & 24 & 8 & 16 & $>0.05$ \\
\hline & WT & 75 & 39 & 36 & \\
\hline & NA & 65 & & & \\
\hline \multirow{3}{*}{ Ki67 exp } & low & 122 & 67 & 55 & 0.049 \\
\hline & high & 17 & 5 & 12 & \\
\hline & NA & 25 & & & \\
\hline \multirow{3}{*}{ MMP9 } & low & 82 & 52 & 30 & 0.001 \\
\hline & high & 82 & 30 & 52 & \\
\hline & NA & 0 & & & \\
\hline \multirow{3}{*}{ PTEN mut } & MUT & 5 & 1 & 4 & $>0.05$ \\
\hline & WT & 90 & 44 & 46 & \\
\hline & NA & 69 & & & \\
\hline \multirow{3}{*}{$1 \mathrm{p} / 19 \mathrm{q}$ co-deletion } & Yes & 32 & 16 & 16 & 1 \\
\hline & No & 132 & 66 & 66 & \\
\hline & NA & 0 & & & \\
\hline \multirow{3}{*}{ EGFR amp } & Yes & 3 & 0 & 3 & $>0.05$ \\
\hline & No & 161 & 82 & 79 & \\
\hline & NA & 0 & & & \\
\hline \multirow{3}{*}{ Pre-operative KPS } & Yes & 45 & 26 & 19 & $>0.05$ \\
\hline & No & 29 & 12 & 17 & \\
\hline & NA & 90 & & & \\
\hline
\end{tabular}

G2: grade 2; G3: grade 3; MT: mutational type; WT: wild type; IDHmut: isocitrate dehydrogenase mutation; TP53: a transcription factor protein which in humans is encoded by the TP53 gene. Ki67: a protein which in humans is encoded by the MKI67 gene; EGFRexp: epidermal growth factor receptor protein expression; MMP9: a migration and invasion marker encoded by the MMP9 protein; PTEN: phosphatase and tensin homolog deleted on chromosome 10. EGFR amp: epidermal growth factor receptor protein amplification; KPS: karnofsky performance score; HR: high-risk; LR: low-risk.

are summarized in Supplementary Table S2. The same $\beta$ value (Table 1) obtained from the CGGA mRNA array set was used to establish a risk score model. Based on the median risk score, we could divide validation sets of lower grade subjects in low- and high-risk groups. Concerning the signature's prognostic value, consistent with the training set, patients with high-risk scores had reduced survival time (Figure 2B and 2C).

\section{Clinical and molecular features of low and high risk lower grade glioma patients}

To assess the relation of current risk groups with previous widely accepted factors and classification systems, basic clinical information and classical molecular markers were analyzed in the CGGA array database. The results are shown in Table 2; there were more patients $(n=12)$ 
Table 3: Univariate and multivariate Cox analysis in CGGA lower grade glioma samples

\begin{tabular}{|c|c|c|c|c|c|c|c|c|}
\hline \multirow{3}{*}{$\begin{array}{l}\text { Clinical } \\
\text { factors }\end{array}$} & \multicolumn{4}{|c|}{ Univariate } & \multicolumn{4}{|c|}{ Multivariate } \\
\hline & \multirow{2}{*}{ HR } & \multicolumn{2}{|c|}{$95 \% \mathrm{CL}$} & \multirow{2}{*}{$\mathbf{P}$} & \multirow{2}{*}{ HR } & \multicolumn{2}{|c|}{$95 \% \mathrm{CL}$} & \multirow{2}{*}{$\mathbf{P}$} \\
\hline & & Lower & Upper & & & Lower & Upper & \\
\hline Histology & 0.564 & 0.316 & 1.006 & 0.05 & 0.581 & 0.169 & 1.997 & 0.388 \\
\hline Grade & 4.309 & 2.419 & 7.676 & $<0.0001$ & 2.262 & 0.751 & 6.81 & 0.147 \\
\hline Risk score & 4.872 & 2.419 & 9.812 & $<0.0001$ & 2.606 & 1.690 & 4.018 & $<0.0001$ \\
\hline $\begin{array}{l}\text { Preoperative } \\
\text { KPS }\end{array}$ & 0.319 & 0.107 & 0.952 & 0.041 & 0.700 & 0.200 & 2.451 & 0.577 \\
\hline
\end{tabular}

Histology: astrocytoma vs oligodendroglioma and oligoastrocytoma; Grade: grade 2 vs grade 3; KPS: karnofsky performance score.

Risk score: high-risk vs low-risk.

Table 4: Univariate and multivariate Cox analysis in TCGA lower grade glioma samples

\begin{tabular}{|c|c|c|c|c|c|c|c|c|}
\hline \multirow{3}{*}{$\begin{array}{l}\text { Clinical } \\
\text { factors }\end{array}$} & \multicolumn{4}{|c|}{ Univatiate } & \multicolumn{4}{|c|}{ Multivariate } \\
\hline & \multirow{2}{*}{ HR } & \multicolumn{2}{|c|}{$95 \% \mathrm{CI}$} & \multirow{2}{*}{$\mathbf{P}$} & \multirow{2}{*}{ HR } & \multicolumn{2}{|c|}{$95 \% \mathrm{CI}$} & \multirow{2}{*}{$\mathbf{P}$} \\
\hline & & Lower & Upper & & & Lower & Upper & \\
\hline Age & 4.153 & 2.65 & 6.507 & $<0.0001$ & 4.330 & 2.645 & 7.088 & $<0.0001$ \\
\hline $\begin{array}{l}\text { IDH1 } \\
\text { mutation }\end{array}$ & 0.344 & 0.226 & 0.524 & $<0.0001$ & 0.591 & 0.376 & 0.929 & 0.023 \\
\hline Gender & 0.98 & 0.654 & 1.468 & 0.921 & & & & \\
\hline Grade & 3.220 & 2.079 & 4.986 & $<0.0001$ & 2.834 & 1.726 & 4.652 & $<0.0001$ \\
\hline Histology & 0.518 & 0.344 & 0.78 & 0.002 & 0.791 & 0.511 & 1.225 & 0.293 \\
\hline Risk score & 2.21 & 1.46 & 3.344 & $<0.0001$ & 3.384 & 2.085 & 5.492 & $<0.0001$ \\
\hline
\end{tabular}

Age: $<41$ vs $\geq 41$; Gender: female vs male; Grade: grade 2 vs grade 3; Histology: astrocytoma vs oligodendroglioma and oligoastrocytoma.

Risk score: high-risk vs low-risk.

with Ki67 overexpression in the high-risk group compared with low-risk individuals $(p=0.049)$. Patients with MMP9 overexpression were more likely found in high-risk group than those not overexpressing this protein $(p=0.001)$. For grade, patients with anaplastic tumors were more likely found in the high-risk group $(p<0.0001)$. Univariate and multivariate Cox regression analyses were further performed in the CGGA mRNA array set (Table 3), and the TCGA RNA-seq database was selected for validation (Table 4). In univariate regression, risk score $(p<0.0001)$ along with grade $(p<0.0001)$ and pre-operative KPS $(p=0.041)$ could promote survival in lower grade gliomas. In multivariate regression, risk score was also significant $(p=0.041)$ after adjusting for grade $(p=0.147)$ and pre-operative KPS $(p=0.119)$. For TCGA RNA-seq set, risk score was also significant $(\mathrm{p}<0.0001)$ in multivariate regression.
Receiver operating characteristic (ROC) assessment was performed to evaluate sensitivity and specificity of two year survival prediction for the threegene signature, histology, grade, age, IDH1 mutation and $1 \mathrm{p} / 19 \mathrm{q}$ co-deletion in the CGGA array dataset (training cohort) and TCGA RNA-seq dataset (the largest validation cohort). We found in the CGGA array dataset the area under the ROC curve (AUROC) for the3-gene set was the largest (0.869). In the TCGA RNA-seq dataset, AUROC value for the 3-gene set (0.785) was slightly smaller than that of age (0.844), but larger than that obtained with IDH1 mutation $(0.759)$ and $1 p / 19 q$ co-deletion (0.593). These results indicated that the three-gene signature might have a better predictive ability in predicting prognosis (Figure 3A-3B). 
Gene function interpretation and expression difference of the three genes in low- and highrisk groups

Little is known concerning the three genes (MN1, HOXA7, and SLC2A4RG) involved in the newly developed signature.
The MN1 gene with two CAG repeat sets is a tumor suppressor. A normal translocation in meningiomas could cause abnormal MN1expression. Meanwhile, MN1 inactivation could induce meningioma formation [15].

The HOXA7 gene belongs to cluster Aon chromosome 7 that controls morphogenesis, organogenesis and differentiation. As a transcription factor, HOXA7

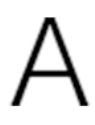

B

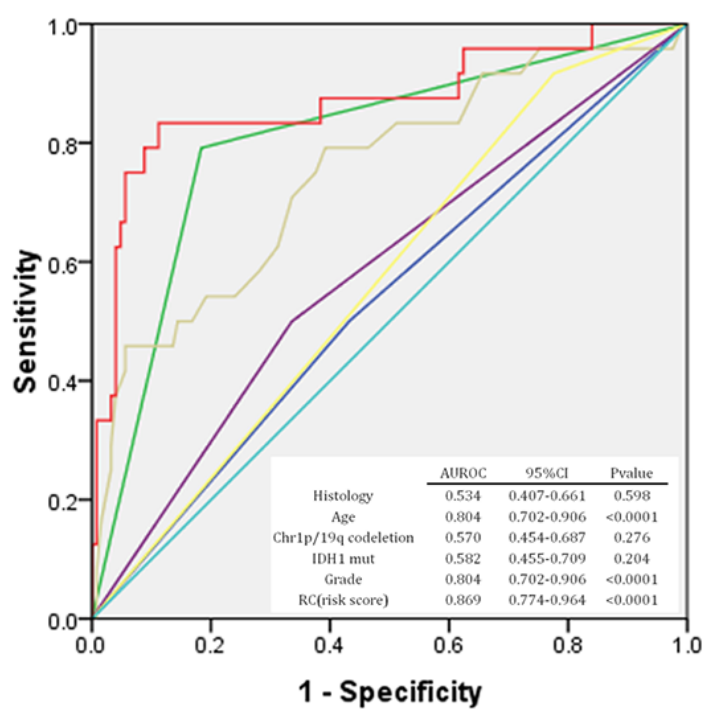

CGGA Roc curve

\section{TCGA Roc curve}

Diagonal segments are produced by ties.

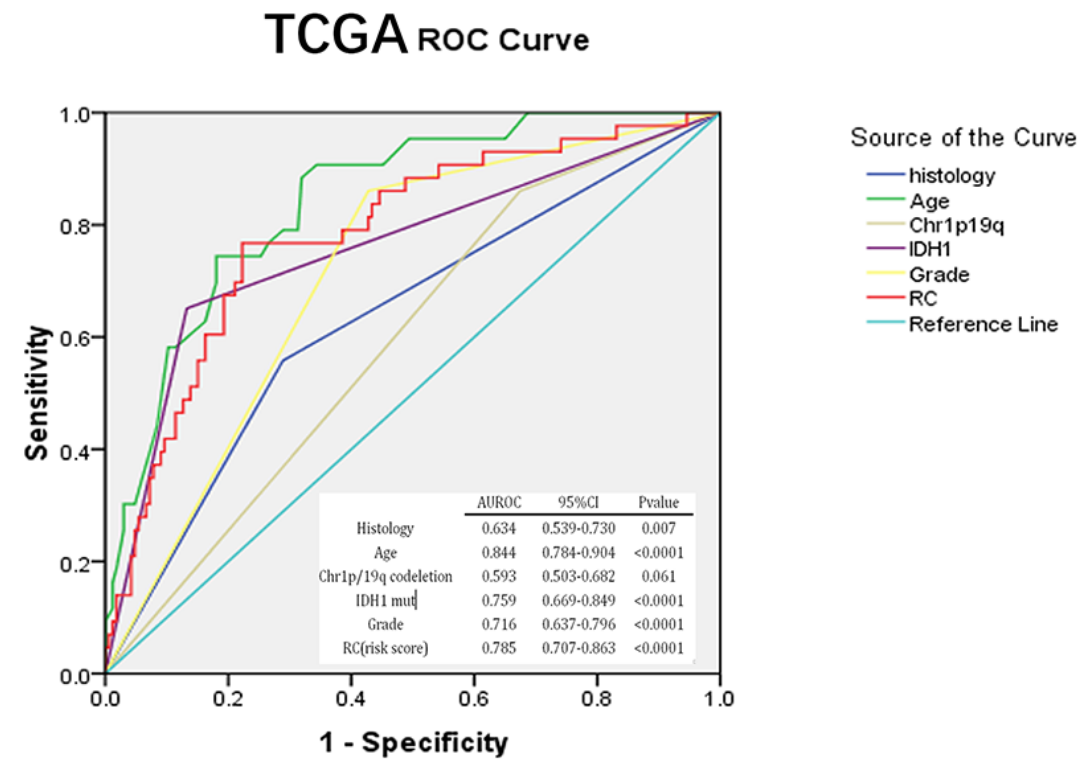

$$
\begin{aligned}
& \text { Source of the Curve } \\
& \text { - histology } \\
& \text { - grade } \\
& \text { - age } \\
& \text { - IDH1 } \\
& \text { Chr1p19q } \\
& \text { - RC } \\
& \text { Reference Line }
\end{aligned}
$$

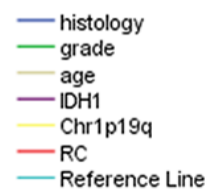

Figure 3: ROC analysis in assessing sensitivity and specificity for two-year survival prediction by the three-gene signature, histology, grade, age, IDH mut, and 1p/19q co-deletion in CGGA mRNA array and TCGA RNA-seq sets (largest validation set). 
regulates many critical genes involved in cancer cell proliferation and invasion [16]. For example, HOXA7 downregulates differentiation-specific genes during keratinocyte proliferation, a repression rescued by differentiation signals. HOXA7is highly similar to antennapedia (Antp) of Drosophila [17].

SLC2A4RG, a transcription factor activates the solute carrier family 2 member 4 gene; it also binds myocyte enhancer factor 2 , another transcription factor, for upregulation of the latter [18].

\begin{tabular}{lcc} 
A NES & NOMp \\
\hline \multicolumn{1}{c}{ Genesets } & N & \\
\hline RECEPTOR_COMPLEX & -1.86 & $<0.0001$ \\
LYMPHOCYTE_ACTIVATION & -1.72 & 0.006 \\
T_CELL_ACTIVATION & -1.70 & 0.010 \\
IMMUNE_SYSTEM_PROCESS & -1.70 & 0.030 \\
DEFENSE_RESPONSE & -1.64 & 0.035 \\
\hline
\end{tabular}

There were marked differences in mRNA amounts between low- and high-risk individuals. Meanwhile, MN1 was overexpressed in the low risk group, with HOXA7 and SLC2A4RG overexpressed in the high risk group (Supplementary Figure S1).

\section{Functional annotation of the signature}

We carried out GSEA and GO analyses to explain the significant OS difference observed between low and high risk score groups; as shown in Figure 4A-4C, T cell

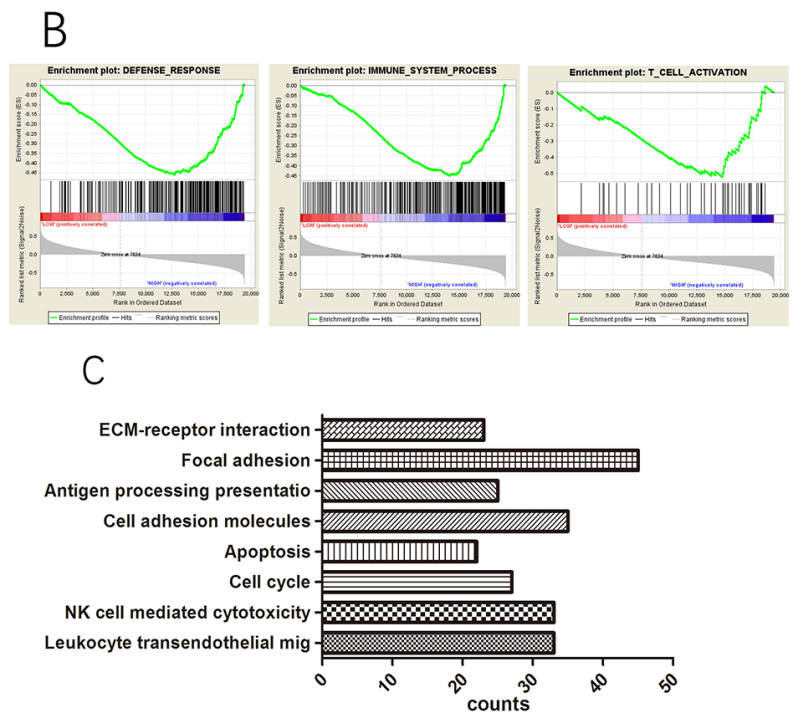

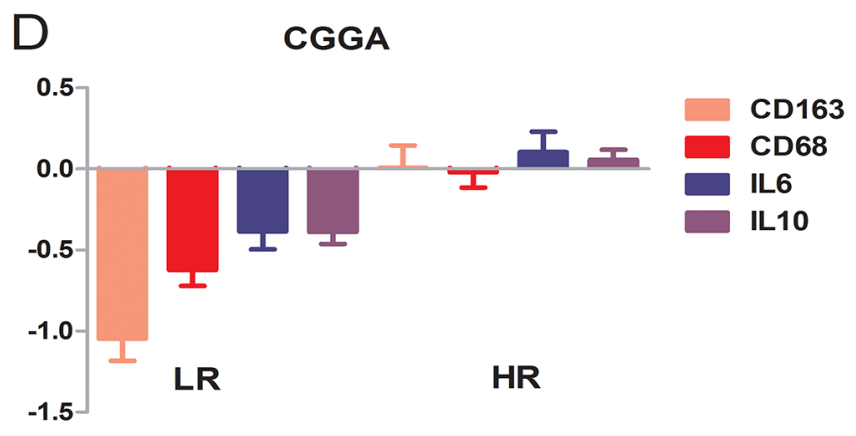

\section{CGGA}

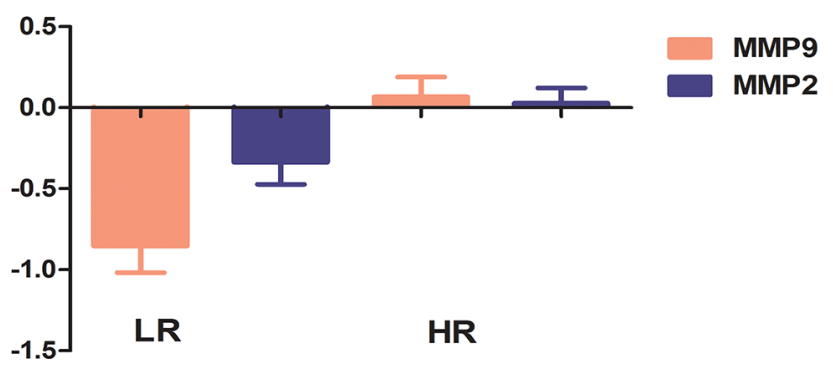

Figure 4: Functional annotation of risk groups. A. Top five enriched pathways in the high-risk group, analysed by gene set enrichment analysis of CGGA data. B. Three representative plots of GSEA from A. C. GO analysis revealed significant associations of genes with increased expression in the high-risk group with eight main pathways. D. Genes encoding CD163, CD68, IL10, IL6, MMP2, and MMP9 are expressed at higher levels in the high-risk group. 
activation as well as receptor complex formation and the immune system process were highly enriched in the high risk group. For GO analysis, cell adhesion, focal adhesion, ECM-receptor interactions, leukocyte trans-endothelial migration, and natural killer cell dependent cytotoxicity were more pronounced in the high-risk group. Such findings may explain prognosis differences between both groups obtained using the signature.

\section{High risk score patients obtained by the three gene signature show tumor associated macrophages (TAMs)- and highly invasive phenotypes}

The results of GSEA and GO analyses revealed that samples in the high-risk score group had TAMs activation and highly invasive phenotypes. in agreement, high-risk group patients showed higher levels of activated M2 macrophage related genes (CD68, CD163, IL10, and IL6, Figure 4D) compared with the low risk group in the training set. Likewise, well-known invasion markers (MMP2 and MMP9) were upregulated in the high-risk group (Figure 4D).

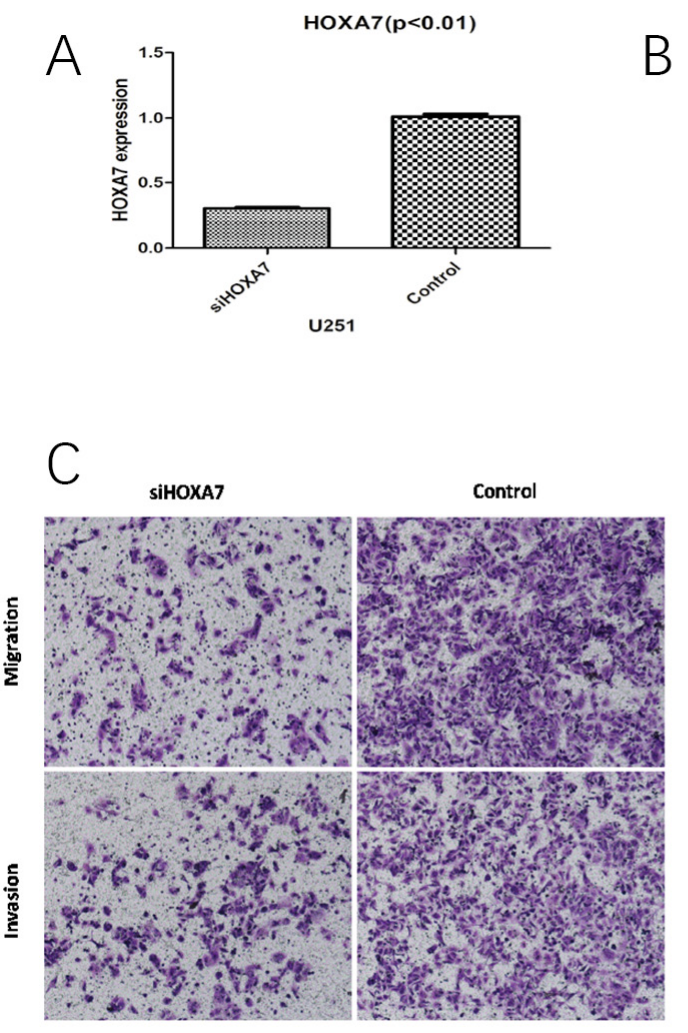

HOXA7-siRNAinhibit migration and invasion in vitro

Studies assessing HOXA7 in glioma are scared. Compared with control siRNA, HOXA7 mRNA and protein levels were considerably reduced in this study (Figure 5A and 5B). In addition, HOXA7-siRNA resulted in the downregulation of MMP9, a well-known migration and invasion marker (Figure 5B). Then, migration and Matrigel invasion assays were carried out using U251 cells. As shown in Figure 5C, HOXA7 knockdown via HOXA7-siRNAclearly inhibited glioma cell migration and invasion (Figure 5C).

\section{DISCUSSION}

Gliomas, which are diagnosed based on histopathological criteria, are among the most frequent and aggressive cerebral tumors. However, the currently available histopathological classification has numerous shortcomings, because glioma is a polygenic and heterogeneous disease, suggesting that accurate molecular classification systems should be developed; this could
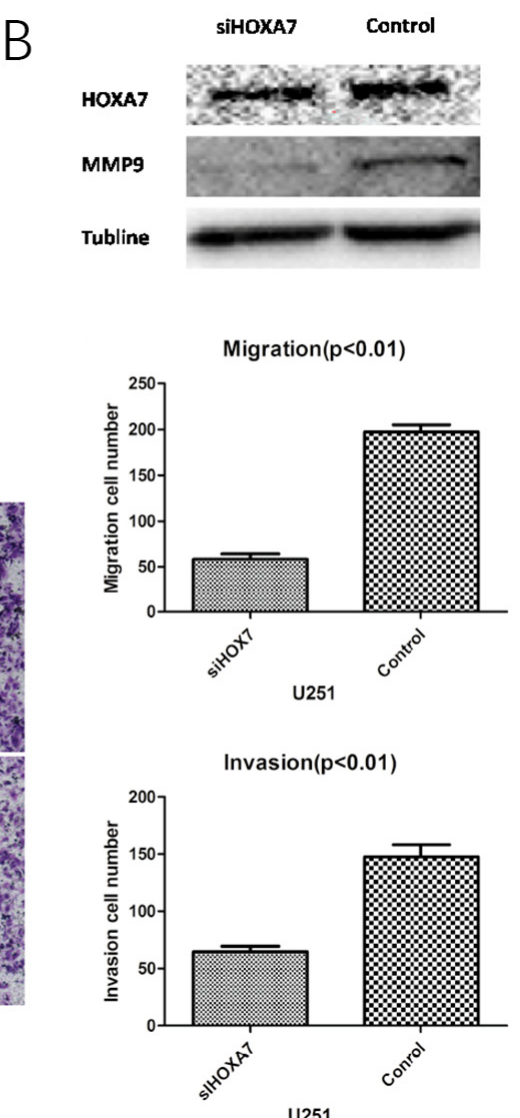

Figure 5: HOXA7 knockdown inhibits glioma cell migration and invasion in vitro. A. RT-qPCR analysis showed that HOXA7siRNA downregulated HOXA7 expression in U251 glioma cells. B. Western blot analysis showed the biological effects of HOXA7-siRNA. Downregulation of HOXA7 inhibited protein expression of MMP9, a well-known migration and invasion marker. C. HOXA7-siRNA inhibited U251 cell migration and invasion capacities $(\mathrm{p}<0.01)$. Representative images and accompanying statistical plots are shown. 
help design more appropriate treatment plans for lower grade glioma cases. Considerable effort has been invested in glioma classification [19, 20], but little is known concerning lower grade gliomas. In this study, a threegene signature (MN1, HOXA7, and SLC2A4RG) could divide lower grade glioma patients into low-and high-risk groups, with longer OS obtained in the former group.

An agreement has not been reached regarding the treatment of lower grade gliomas. Previous findings demonstrated that chemotherapy does not prolong OS in patients with isocitrate dehydrogenase 1-mutant (IDH1 mut) tumors in low grade gliomas [21]. Meanwhile, RTOG showed that combining PCV chemotherapy and radiotherapy improves median OS of patients with grade II gliomas who received no total tumor resection or were $>40$ years old [22]. The identified three-gene signature could provide an objective and accurate classification model. Furthermore, based on the three-gene signature, suitable therapies could be selected for different lower grade glioma patients. More aggressive therapeutic regimens could be used for high-risk patients, avoiding overtreatment of low-risk group patients.

In glioma mass, TAMs induced by glioma cells could provide a supportive microenvironment for tumor cell proliferation and invasion [23]. The high-risk group showed the TAMs phenotype, with higher levels of IL10, IL6, CD68 and CD163, classical markers for M2 TAMs [24]. Meanwhile, the expression levels of MMP9 and MMP2 were higher in high-risk patients compared with the low-risk group, suggesting gliomas had increased invasion potential in the former group. In the high-risk score group, there were more activated M2 TAMs than in low-risk patients, which may explain why gliomas in the high-risk group had increased invasion ability.

In summary, a three-gene signature was used to divide lower grade gliomas into low- and high-risk groups. High-risk group samples induced more M2 TAMs and had increased invasion potential. This signature constitutes a new prognostic model for classifying lower grade glioma patients more objectively and accurately compared with current methods, and could improve patient's quality of life.

\section{MATERIALS AND METHODS}

\section{Datasets and patients}

Samples were obtained from the Chinese Glioma Genome Atlas (CGGA) dataset (the training set); the TCGA RNA-seq database and GSE16011 array dataset were assessed as independent validation sets. Overall survival (OS) time (the period from surgery to death) was obtained through phone interviews with the patients and/ or their relatives. Patients with survival shorter than 1year were considered short OS patients; those surviving for more than 5 years were classified in the long OS group.

\section{Real-time polymerase chain reaction (RT-PCR) and Western blot}

RT-PCR and Western blot were carried out as recently described $[25,26]$. RT-PCR primers were: HOXA7-F, 5'- TCGTATTATGTGAACGCGCTT-3' and HOXA7-R, 5'-CAAGAAGTCGGCTCGGCATT -3'; GAPDH was used as a reference gene. Data were analyzed by the $2-\Delta \Delta \mathrm{Ct}$ method. In Western blot experiments, TUBLINE was employed as a loading control.

\section{Cell culture and transfection of HOXA7-siRNA}

Human glioma U251 cells were obtained from the American Type Culture Collection (ATCC), and maintained in DMEM containing $10 \%$ fetal bovine serum (FBS; Gibco, USA) and antibiotics (100 U/ml penicillin and $100 \mu \mathrm{g} / \mathrm{mL}$ streptomycin) in a humid environment with $5 \% \mathrm{CO} 2$ at $37^{\circ} \mathrm{C}$. HOXA7-siRNAs (sense, 5'-CAAACUACCUAUGUAUCCATT-3'; antisense, 5'-UGGAUACAUAGGUAGUUUGTT-3') and a non-specific control siRNA (NC) were purchased from Genepharma (Shanghai, China), and transfected into U251 cells using Lipofectamine ${ }^{\mathrm{TM}} 2000$ (Invitrogen, Shanghai, China). U251 cells were infected with HOXA7-siRNA for $48 \mathrm{~h}$ before RT-PCR and Western blot assays [27].

\section{Gene set enrichment analysis (GSEA)}

The Gene Set Enrichment Analysis software was used to perform GSEA. Based on median risk scores, the patients were divided into two groups: low- and highrisk groups. Then, ontology gene set from MSigDB was performed to assess enrichment gene sets for the threegene signature.

\section{Statistical analyses}

Among the 164 lower grade glioma specimens, 117 grade II and 47 grade III samples were identified. These included 13 cases whose survival times were below 1 year and 17 who survived for more than 5 years. T-test and FDR correction were performed to compare differentially expressed genes between these two groups. FDR $<0.05$ was considered statistically significant. Univariate Cox regression analysis was carried out to assess these genes in predicting patient survival. A total of 1347 and 4224 genes showed significant differences $(p<0.05)$ in grade II and III patients, respectively. The differentially expressed genes common in both groups were validated by univariate Cox analysis in TCGA RNA-seq and GSE 16011 array databases. Three genes were adopted to establish the gene signature model (Figure 6). To assess the clinical value of the three-gene signature in predicting OS, a mathematical was generated. Specifically, as proposed previously [8], every patient was attributed a risk score based on 
linear combination of mRNA levels and Cox regression coefficients in lower grade glioma for the three genes: $\times \beta_{\text {gene3 }}$

Risk score $=\operatorname{expr}_{\text {gene } 1} \times \beta_{\text {gene } 1+} \operatorname{expr}_{\text {gene2 }} \times \beta_{\text {gene } 2+} \operatorname{expr}_{\text {gene } 3}$

Patients with no recorded survival time were excluded. For a gene with multiple probes, average expression was used to calculate the risk score. OS in high- and low-risk patient groups was estimated by the Kaplan-Meier method and 2-sided log-rank test in GraphPad Prism Version 6.01. Multiple Cox regression analysis was used to assess independent contributions to OS prediction after adjusting for other classical clinicopathologic variables [28].

\section{ACKNOWLEDGMENTS}

We thank Yuling Yang for the collection of tissue samples and the retrieval of clinical data. This research was funded by the Research Special Fund for Public Welfare Industry of Health (No. 201402008), the National High Technology Research and Development Program (No. 2012AA02A508), the International Science and Technology Cooperation Program (No. 2012DFA30470), the National Natural Science Foundation of China (No. 91229121), the Beijing Science and Technology Plan (No. Z131100006113018), the National Key Technology Research and Development Program of the Ministry of

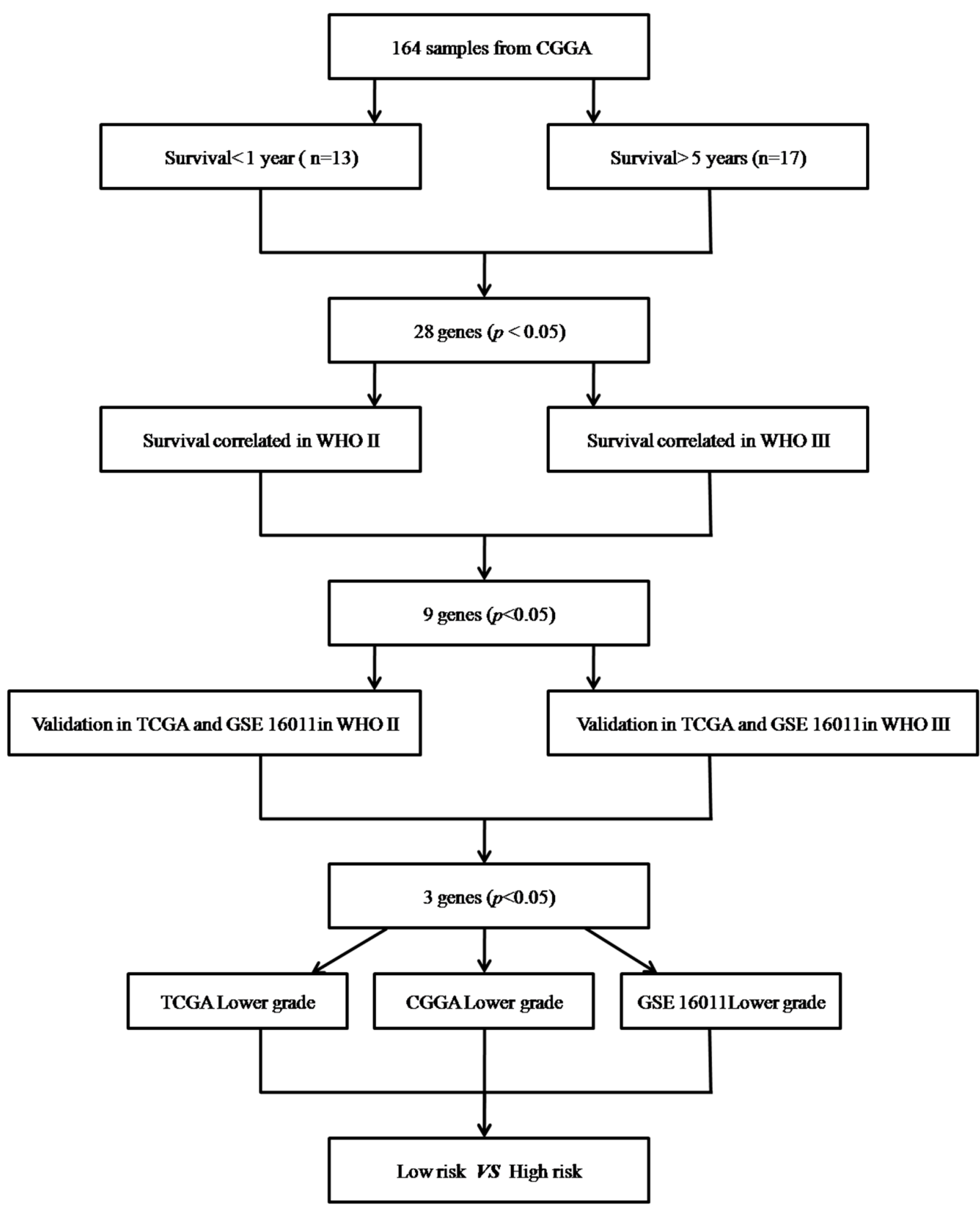

Figure 6: Procedure for selecting the 3 genes (HOXA7, SLC2A4RG and MN1) through whole genome mRNA profiling. 
Science and Technology of China (No. 2013BAI09B03 and 2014BAI04B02).

\section{CONFLICTS OF INTEREST}

None for all authors.

\section{FUNDING}

This study was supported by funds from the National Nature Science Foundation of China (grant no. 81301112).

\section{REFERENCES}

1. Gao KM, Chen XC, Zhang JX, Wang Y, Yan W, You YP. A pseudogene-signature in glioma predicts survival. Journal of experimental \& clinical cancer research. 2015; 34:23.

2. Wang Y, Wang X, Zhang J, Sun G, Luo H, Kang C, Pu P, Jiang T, Liu N, You Y. MicroRNAs involved in the EGFR/ PTEN/AKT pathway in gliomas. Journal of neuro-oncology. 2012; 106:217-224.

3. Yang P, You G, Zhang W, Wang Y, Wang Y, Yao K, Jiang T. Correlation of preoperative seizures with clinicopathological factors and prognosis in anaplastic gliomas: a report of 198 patients from China. Seizure. 2014; 23:844-851.

4. Chan AK, Yao Y, Zhang Z, Shi Z, Chen L, Chung NY, Liu JS, Li KK, Chan DT, Poon WS, Wang Y, Zhou L, Ng HK. Combination genetic signature stratifies lower-grade gliomas better than histological grade. Oncotarget. 2015; 6:20885-20901. doi: 10.18632/oncotarget.4928.

5. Jiang T, Mao Y, Ma W, Mao Q, You Y, Yang X, Jiang C, Kang C, Li X, Chen L, Qiu X, Wang W, Li W, Yao Y, Li $\mathrm{S}$, Li S, et al. CGCG clinical practice guidelines for the management of adult diffuse gliomas. Cancer letters. 2016; 375:263-273.

6. Agnihotri S, Aldape KD, Zadeh G. Isocitrate dehydrogenase status and molecular subclasses of glioma and glioblastoma. Neurosurgical focus. 2014; 37:E13.

7. Ludwig JA, Weinstein JN. Biomarkers in cancer staging, prognosis and treatment selection. Nature reviews Cancer. 2005; 5:845-856.

8. Zhang W, Zhang J, Yan W, You G, Bao Z, Li S, Kang C, Jiang C, You Y, Zhang Y, Chen CC, Song SW, Jiang T. Whole-genome microRNA expression profiling identifies a 5-microRNA signature as a prognostic biomarker in Chinese patients with primary glioblastoma multiforme. Cancer. 2013; 119:814-824.

9. Dietrich D, Kneip C, Raji O, Liloglou T, Seegebarth A, Schlegel T, Flemming N, Rausch S, Distler J, Fleischhacker M, Schmidt B, Giles T, Walshaw M, Warburton C, Liebenberg V, Field JK. Performance evaluation of the DNA methylation biomarker SHOX2 for the aid in diagnosis of lung cancer based on the analysis of bronchial aspirates. International journal of oncology. 2012; 40:825-832.

10. Phan B, Majid S, Ursu S, de Semir D, Nosrati M, Bezrookove V, Kashani-Sabet M, Dar AA. Tumor suppressor role of microRNA-1296 in triple-negative breast cancer. Oncotarget. 2016; 7:19519-19530. doi: 10.18632/ oncotarget.6961.

11. Labussiere M, Boisselier B, Mokhtari K, Di Stefano AL, Rahimian A, Rossetto M, Ciccarino P, Saulnier O, Paterra R, Marie Y, Finocchiaro G, Sanson M. Combined analysis of TERT, EGFR, and IDH status defines distinct prognostic glioblastoma classes. Neurology. 2014; 83:1200-1206.

12. Tabouret E, Bequet C, Denicolai E, Barrie M, Nanni I, Metellus P, Dufour H, Chinot O, Figarella-Branger D. BRAF mutation and anaplasia may be predictive factors of progression-free survival in adult pleomorphic xanthoastrocytoma. European journal of surgical oncology. 2015; 41:1685-1690.

13. Liu Y, Hu H, Wang K, Zhang C, Wang Y, Yao K, Yang P, Han L, Kang C, Zhang W, Jiang T. Multidimensional analysis of gene expression reveals TGFB1I1-induced EMT contributes to malignant progression of astrocytomas. Oncotarget. 2014; 5:12593-12606. doi: 10.18632/oncotarget.2518.

14. Franceschini A, Szklarczyk D, Frankild S, Kuhn M, Simonovic M, Roth A, Lin J, Minguez P, Bork P, von Mering C, Jensen LJ. STRING v9.1: protein-protein interaction networks, with increased coverage and integration. Nucleic acids research. 2013; 41:D808-815.

15. Bosson C, Devillard F, Satre V, Dieterich K, Ray PF, Morand B, Dubois-Teklali F, Vieville G, Andrieux J, Brouillet S, Amblard F, Jouk PS, Coutton C. Microdeletion $\operatorname{del}(22)(q 12.1)$ excluding the MN1 gene in a patient with craniofacial anomalies. American journal of medical genetics Part A. 2015.

16. Li Y, Yang XH, Fang SJ, Qin CF, Sun RL, Liu ZY, Jiang BY, Wu X, Li G. HOXA7 stimulates human hepatocellular carcinoma proliferation through cyclin E1/CDK2. Oncology reports. 2015; 33:990-996.

17. Di Vinci A, Brigati C, Casciano I, Banelli B, Borzi L, Forlani A, Ravetti GL, Allemanni G, Melloni I, Zona G, Spaziante R, Merlo DF, Romani M. HOXA7, 9, and 10 are methylation targets associated with aggressive behavior in meningiomas. Translational research. 2012; 160:355-362.

18. Song X, Zhou K, Zhao Y, Huai C, Zhao Y, Yu H, Chen Y, Chen G, Chen H, Fan W, Mao Y, Lu D. Fine mapping analysis of a region of $20 \mathrm{q} 13.33$ identified five independent susceptibility loci for glioma in a Chinese Han population. Carcinogenesis. 2012; 33:1065-1071.

19. Leeper HE, Caron AA, Decker PA, Jenkins RB, Lachance DH, Giannini C. IDH mutation, 1p19q codeletion and ATRX loss in WHO grade II gliomas. Oncotarget. 2015; 6:30295-30305. doi: 10.18632/oncotarget.4497.

20. Yan W, Zhang W, You G, Zhang J, Han L, Bao Z, Wang Y, Liu Y, Jiang C, Kang C, You Y, Jiang T. Molecular 
classification of gliomas based on whole genome gene expression: a systematic report of 225 samples from the Chinese Glioma Cooperative Group. Neuro-oncology. 2012; 14:1432-1440.

21. Li MY, Wang YY, Cai JQ, Zhang CB, Wang KY, Cheng W, Liu YW, Zhang W, Jiang T. Isocitrate dehydrogenase 1 Gene Mutation Is Associated with Prognosis in Clinical Low-Grade Gliomas. PloS one. 2015; 10:e130872.

22. van den Bent MJ. Practice changing mature results of RTOG study 9802: another positive PCV trial makes adjuvant chemotherapy part of standard of care in lowgrade glioma. Neuro-oncology. 2014; 16:1570-1574.

23. Hambardzumyan D, Gutmann DH, Kettenmann H. The role of microglia and macrophages in glioma maintenance and progression. Nature neuroscience. 2015; 19:20-27.

24. Prosniak M, Harshyne LA, Andrews DW, Kenyon LC, Bedelbaeva K, Apanasovich TV, Heber-Katz E, Curtis MT, Cotzia P, Hooper DC. Glioma grade is associated with the accumulation and activity of cells bearing M2 monocyte markers. Clinical cancer research. 2013; 19:3776-3786.
25. You G, Feng L, Yan W, Zhang W, Wang YZ, Li SW, Li SW, Li GL, Song YJ, Kang CS, You YP, Jiang T. BCL2A1 is a potential biomarker for postoperative seizure control in patients with low-grade gliomas. CNS Neurosci Ther. 2013; 19:882-8.

26. Li MY, Yang P, Liu YW, Zhang CB, Wang KY, Wang YY, Yao K, Zhang W, Qiu XG, Li WB, Peng XX, Wang YZ, Jiang T. Low c-Met expression levels are prognostic for and predict the benefits of temozolomide chemotherapy in malignant gliomas. Scientific reports. 2016; 6:21141.

27. Wang X, Shi W, Shi H, Lu S, Wang K, Sun C, He J, Jin W, Lv X, Zou H, Shu Y. TRIM11 overexpression promotes proliferation, migration and invasion of lung cancer cells. Journal of experimental \& clinical cancer research. 2016; 35:100.

28. Zhang W, Zhang J, Hoadley K, Kushwaha D, Ramakrishnan V, Li S, Kang C, You Y, Jiang C, Song SW, Jiang T, Chen CC. miR-181d: a predictive glioblastoma biomarker that downregulates MGMT expression. Neuro-oncology. 2012; 14:712-719. 